\title{
EXPERIMENTAL EVIDENCE FOR MULTI-BAND CONDUCTION IN HIGHLY-DOPED La-Sr-Cu-O SUPERCONDUCTORS
}

\author{
C. UHER ${ }^{1}$ \\ Max-Planck-Institut für Festkörperforschung, Postfach 8006 65, 7000 Stuttgart 80, FRG \\ and Physics Department, The University of Michigan, Ann Arbor, MI 48109, USA
}

and

\author{
A.B. KAISER ' \\ Max-Planck-Institut für Festkörperforschung, Postfach 8006 65, 7000 Stuttgart 80, FRG
}

Received 13 August 1987; accepted for publication 2 October 1987

Communicated by D. Bloch

\begin{abstract}
We have measured the Hall coefficient in highly-doped $\mathrm{La}_{2-x} \mathrm{Sr}_{x} \mathrm{CuO}_{4}$ superconductors $(x=0.2$ and 0.25$)$, finding a large decrease with temperature. A good fit to the data is obtained using the two-band model involving both holes and electrons (as suggested by our earlier thermopower measurements), with the carrier-phonon interaction being relatively stronger for the holes.
\end{abstract}

Much insight has been gained over the past few months into the physical properties of the recently discovered high $T_{\mathrm{c}}$ superconductors based on the oxygen-deficient perovskites. To help elucidate the focal point, namely the nature of the coupling mechanism responsible for the unprecedentedly high $T_{\mathrm{c}}$ observed in this class of materials, a wide range of experimental techniques has been brought to bear on this problem. Pivotal among these studies are investigations of the normal state behavior, which provide fundamental input parameters for various theories of superconductivity. Perhaps potentially the most profitable among these are measurements of the Hall coefficient, which yield not only an estimate of the carrier density and identify the polarity of the dominant carriers, but also point out, via the temperature dependence, any unusual carrier scattering features and multiplicity of the carrier spectrum. While in principle the Hall effect measurements are very simple, the extremely small mobilities of a few $\mathrm{cm}^{2} \mathrm{~V}^{-1} \mathrm{~s}^{-1}$ typical of these ceramics result in very small Hall voltages that have to be carefully dis-

\footnotetext{
I Alexander von Humboldt Fellow.
}

cerned from a large and strongly temperature dependent resistive voltage. This complicates the measurements and the available data on the temperature dependence are, so far, by no means plentiful.

Panson et al. [1] measured a Hall coefficient of magnitude $(5 \pm 2) \times 10^{-9} \mathrm{~m}^{3} \mathrm{C}^{-1}$ on a sample of $\mathrm{La}-\mathrm{Sr}-\mathrm{Cu}-\mathrm{O}(x=0.2)$ at $48 \mathrm{~K}$ in a field of $5 \mathrm{~T}$. Most surprisingly, these authors report the Hall constant as negative, contrary to an intuitive picture of holedominated transport and subsequent Hall effect investigations. Ong et al. [2] reported a strong compositional dependence of the Hall effect measured at $77 \mathrm{~K}$ on a series of $\mathrm{La}-\mathrm{Sr}-\mathrm{Cu}-\mathrm{O}$ compounds spanning the $\mathrm{Sr}$ content range between $x=0.05$ and 0.25 . They noted a precipitous fall in the Hall constant setting in at $x=0.15$, and stated that above $x=0.18$ the signal became undetectably small in spite of a $15 \mathrm{~T}$ transverse field used in these studies. Hundley et al. [3] made investigations on a sample of $\mathrm{La}-\mathrm{Sr}-\mathrm{Cu}-\mathrm{O}$ $(x=0.15)$ which indicate a linearly decreasing Hall constant with increasing temperature. In addition, they noted an interesting sharp "spike" on the data just as the sample goes superconducting, which they 
ascribe to an enhanced magnetic field in the normal regions of the sample as the flux is excluded from the superconducting grains. A modest temperature dependence of the Hall coefficient in $\mathrm{La}-\mathrm{Sr}-\mathrm{Cu}-\mathrm{O}$ for $x=0.15$ and $x=0.185$ was also found by Penney et al. [4].

In the course of our recent studies on the electronic and thermal transport properties of $\mathrm{La}-\mathrm{Sr}-\mathrm{Cu}-\mathrm{O}$ superconductors [5] we have observed a sign change (from positive to negative) in the thermopower of the most heavily Sr-doped compounds with increasing temperature and we have pointed out the likelihood of a multicarrier nature of the carrier spectrum in these materials. To substantiate this point we have measured the temperature dependence of the Hall coeffient on these samples and we describe the results in this Letter.

dc Hall measurements were made on samples of $\mathrm{La}-\mathrm{Sr}-\mathrm{Cu}-\mathrm{O}$ with $x=0.2$ and $x=0.25$ cut from the same disks as used for the thermopower studies. The samples were thinned down to about $0.25 \mathrm{~mm}$ thickness and copper leads were attached with the aid of silver epoxy. Samples were coated with Apiezon grease and fastened with a dental floss to a cigarette paper-insulated copper cold tip of a cryostat. The five-probe (resistive voltage cancellation) as well as four-probe technique with a field reversal were employed and gave comparable results. The data were taken with a current density of $0.5 \mathrm{~A} \mathrm{~cm}^{-2}$ in a field of $7 \mathrm{~T}$. To make sure that the temperature of the samples is the same after the field is reversed from +7 to $-7 \mathrm{~T}$ (about 10 minutes procedure) a small, flat $\mathrm{Pt}$ thermometer was attached to each sample using Apiezon grease. While Pt has a substantial magnetoresistance, at a fixed field its resistance is immune to the field reversal. The base temperature was monitored by a calibrated glassy-carbon sensor.

Our results are shown in fig. 1 , together with the earlier data [3] for a $x=0.15$ sample. It is clear that the trend of the two data sets is consistent. What our new data show is that besides reducing in size as $x$ increases, the Hall coefficient $R_{\mathrm{H}}$ shows a much stronger relative temperature dependence. For the $x=0.25$ sample, the decrease in $R_{\mathrm{H}}$ between 40 and $200 \mathrm{~K}$ is nearly $70 \%$. These measurements provide evidence that the widely used one-band model is not applicable for the interpretation of the Hall effect at least in the highly doped $\mathrm{La}-\mathrm{Sr}-\mathrm{Cu}-\mathrm{O}$ supercon-

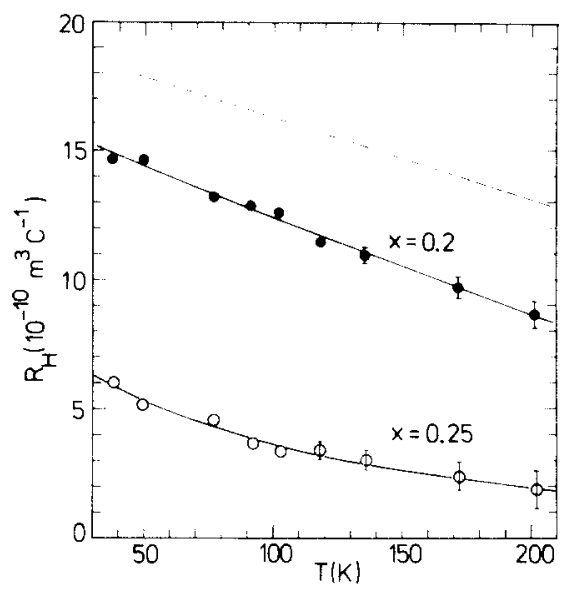

Fig. 1. Our measurements (circles) of the Hall coefficient in $\mathrm{La}_{2-x} \mathrm{Sr}_{x} \mathrm{CuO}_{4}$ with $x=0.2$ and 0.25 , with the results of Hundley et al. [3] for $x=0.15$ for comparison (dotted line). The full lines are calculations for the two-band model with parameters $\rho_{\mathrm{h}}(100)=540 \mu \Omega \mathrm{cm}, \rho_{\mathrm{h}}(200)=1030 \mu \Omega \mathrm{cm}, \rho_{\mathrm{e}}(100)=4090 \mu \Omega$ $\mathrm{cm}, \quad \rho_{\mathrm{e}}(200)=4280 \mu \Omega \mathrm{cm}, \quad n_{\mathrm{h}}=3.7 \times 10^{21} \mathrm{~cm}^{-3}$, and $n_{\mathrm{c}}=1.0 \times 10^{21} \mathrm{~cm}^{-3}$ for the $x=0.2$ sample, and $\rho_{\mathrm{h}}(100)=720 \mu \Omega$ $\mathrm{cm}, \quad \rho_{\mathrm{h}}(200)=1280 \mu \Omega \quad \mathrm{cm}, \quad \rho_{\mathrm{c}}(100)=1660 \mu \Omega \quad \mathrm{cm}$, $\rho_{\mathrm{c}}(200)=2360 \mu \Omega \mathrm{cm}, n_{\mathrm{b}}=5.2 \times 10^{21} \mathrm{~cm}^{-3}$, and $n_{\mathrm{c}}=2.5 \times 10^{21}$ $\mathrm{cm}^{-3}$ for the $x=0.25$ sample

ductors, and that it is not possible in these cases to obtain reliable values for the density of carriers using this model.

In strong contrast to the case of pure $\mathrm{La}_{2} \mathrm{CuO}_{4}$ [6] and lightly Sr-doped compounds [7], the transport properties for $\mathrm{La}-\mathrm{Sr}-\mathrm{Cu}-\mathrm{O}$ with $x$ greater than 0.15 are consistent with those expected for ordinary metallic conduction but with a small density of carriers [5]. It is, of course, likely that the electrons in these superconductors are moderately strongly correlated $[8,9]$, but the correlations do not appear strong enough to change the linear metallic resistivity dependence, for example. It therefore seems worthwhile to attempt to account for the Hall coefficient behaviour in terms of the usual metallic two-band expression [10]

$R_{\mathrm{H}}=\left(\frac{\rho}{\rho_{\mathrm{h}}}\right)^{2} \frac{1}{n_{\mathrm{h}} e}-\left(\frac{\rho}{\rho_{\mathrm{e}}}\right)^{2} \frac{1}{n_{\mathrm{e}} e}$.

Here $n_{\mathrm{h}}$ and $n_{\mathrm{e}}$ are the carrier densities (assumed independent of temperature $T$ ) for the hole and electron bands respectively, $e$ is the magnitude of electronic charge, $\rho_{\mathrm{h}}$ and $\rho_{\mathrm{e}}$ are the corresponding re- 
sistivities for the two bands, and $\rho$ is the total resistivity

$\rho=\rho_{\mathrm{h}} \rho_{\mathrm{e}} /\left(\rho_{\mathrm{h}}+\rho_{\mathrm{e}}\right)$.

The band resistivities $\rho_{\mathrm{h}}$ and $\rho_{\mathrm{e}}$ are taken as linear in the temperature range of our Hall measurements (approximately 40 to $200 \mathrm{~K}$ ); we write them in the form

$$
\begin{aligned}
\rho_{\mathrm{i}} & =(2-T / 100) \rho_{i}(100)-(1-T / 100) \rho_{i}(200) \\
& (i=\mathrm{h}, \mathrm{e}),
\end{aligned}
$$

since it is convenient to characterize the resistivities $\rho_{i}$ by their values $\rho_{i}(100)$ and $\rho_{i}(200)$ at temperatures 100 and $200 \mathrm{~K}$ respectively. The observed values of the total resistivity $\rho$ at these temperatures provide two constraints for the four parameters $\rho_{i}(100)$ and $\rho_{i}(200)$, but since both the observed and calculated resistivity (for the parameter ranges of interest) are approximately linear, two degrees of freedom remain.

As shown by the examples in fig. 1, the two-band model can give an excellent account of the data for reasonable parameter values. Even the sign of the curvature apparent in the $x=0.25$ data is consistent with the two-band model prediction. Since the values of the parameters are not uniquely determined by the data, the precise values for $n_{\mathrm{h}}$ and $n_{\mathrm{e}}$ are not significant. However, the essential features required for the two-band model to fit our experimental data are as follows.

Because $R_{\mathrm{H}}$ is positive, holes are the dominant carriers, with a density of a few times $10^{21} \mathrm{~cm}^{-3}$. The temperature dependence of $R_{\mathrm{H}}$ in our calculation arises because the contribution from the electron band is weighted more strongly as temperature increases, i.e. because $\rho_{\mathrm{h}}$ for the hole band increases with $T$ relatively faster than $\rho_{\mathrm{e}}$ for the electron band; for example, we find $\rho_{\mathrm{h}}(200) / \rho_{\mathrm{h}}(100)=1.79$ compared to $\rho_{\mathrm{e}}(200) / \rho_{\mathrm{e}}(100)=1.42$ for the parameters pertaining to the $x=0.25$ curve in fig. 1 , and 1.89 and 1.05 respectively for the parameters for the $x=0.2$ sample. Such behavior arises if the coupling between the holes and phonons is much stronger than that between electrons and phonons, leading to a faster increase with $T$ of $\rho_{\mathrm{h}}$ than $\rho_{\mathrm{e}}$ relative to the corresponding "residual" resistivities (a smaller structural scattering of the holes could also contribute to this effect). A strong interaction of holes and phonons is consistent with our earlier conclusion [5] from the size of the temperature dependence of resistivity. The effect of the electrons is clearly more significant for $x=0.25$ than for the lower concentrations, indicating that the density of electrons increases faster with $\mathrm{Sr}$ concentration $x$ than that of holes.

For the parameter values listed in the caption to fig. 1 , and for $\rho_{\mathrm{h}}$ and $\rho_{\mathrm{e}}$ assumed linear in the temperature range of interest, the total resistivity $\rho$ shows a very slight downward curvature below a linear law at lower temperatures. Such behavior, however, would likely be masked in practice by the upward curvature of the electron-phonon contribution that typically occurs below about $50 \mathrm{~K}$. Such an upward curvature does appear to be observed [5] for the $x=0.25$ sample with the lower $T_{\mathrm{c}}$ value, but superconducting precursor effects soon produce a large decrease in $\rho$ as $T$ decreases.

In conclusion, we have demonstrated strong evidence for multi-band conduction in highly doped $\mathrm{La}-\mathrm{Sr}-\mathrm{Cu}-\mathrm{O}$. The fact that our present and previous data [5] are explicable in terms of usual metallic conduction with a strong hole-phonon interaction without the need for exotic mechanisms suggests that this hole-phonon coupling could at least play a role in the origin of the high $T_{\mathrm{c}}$ superconductivity.

We wish to thank our respective hosts, Professors E. Gmelin and P. Fulde, and members of the laboratory for their hospitality and support, and Dr. L. Walz for making available the samples of La-Sr-CU-O. We acknowledge the support of Fellowships from the Alexander von Humboldt- Stiftung. This work was supported in part by the NSF - Low Temperature Physics - Grant DMR-8508392.

\section{References}

[1] A.J. Panson, G.R. Wagner, A.I. Braginski, J.R. Gavaler, M.A. Janocko, H.C. Pohl and J. Talvacchio, Appl. Phys. Lett. 50 (1987) 1104.

[2] N.P. Ong, Z.Z. Wang, J. Clayhold, J.M. Tarascon, L.H Greene and W.R. McKinnon, Phys. Rev. B 35 (1987) 8807.

[3] M.F. Hundley, A. Zettl, A. Stacey and M.L. Cohen, Phys. Rev. B 35 (1987) 8800.

[4] T. Penney, M.W. Schafer, B.L. Olson and T.S. Plaskett, J. Am. Ceram. Soc., to be published. 
[5] C. Uher, A.B. Kaiser, E. Gmelin and L. Walz, preprint.

[6] P.M. Grant, S.S.P. Parkin, V.Y. Lee, E.M. Engler, M.L. Ramirez, J.E. Vazquez, G. Lim, R.D. Jacowitz and R.L. Greene, Phys. Rev. Lett. 58 (1987) 2482.

[7] J.R. Cooper, B. Alavi, L.-W. Zhou, W.P. Beyermann and G. Grüner, Phys. Rev. B 35 (1987) 8794.
[8] R.L. Greene, H. Maletta, T.S. Plaskett, J.G. Bednorz and K.A. Müller, Solid State Commun. 63 (1987) 379.

[9] A.M. Oles, J. Zaanen and P. Fulde, preprint.

[10] FJ. Blatt, Physics of electronic conduction in solids (McGraw-Hill, New York, 1968). 